\title{
A Hybrid 3D Indoor Space Model
}

\author{
Ali Jamali ${ }^{1}$, Alias Abdul Rahman ${ }^{1}$ and Pawel Boguslawski ${ }^{2}$ \\ ${ }^{1}$ Universiti Teknologi Malaysia (UTM) \\ Faculty of Geoinformation and Real Estate \\ ali.jamali.65@gmail.com, \\ alias@utm.my \\ ${ }^{2}$ University of the West of England \\ Faculty of Environment and Technology \\ Pawel.boguslawski@uwe.ac.uk
}

KEY WORDS: 3D Data Modeling, Indoor Navigation, Topology

\begin{abstract}
GIS integrates spatial information and spatial analysis. An important example of such integration is for emergency response which requires route planning inside and outside of a building. Route planning requires detailed information related to indoor and outdoor environment. Indoor navigation network models including Geometric Network Model (GNM), Navigable Space Model, sub-division model and regular-grid model lack indoor data sources and abstraction methods. In this paper, a hybrid indoor space model is proposed. In the proposed method, 3D modeling of indoor navigation network is based on surveying control points and it is less dependent on the 3D geometrical building model. This research proposes a method of indoor space modeling for the buildings which do not have proper 2D/3D geometrical models or they lack semantic or topological information. The proposed hybrid model consists of topological, geometrical and semantical space.
\end{abstract}

\section{Introduction}

Navigation in indoor space is limited by walls, doors and obstacles (e.g. furniture). According to Zhang et al. (2016), geometric model, symbolic model, object feature model, and hybrid model are existing indoor space models. Geometric model focuses on the geometric represention of indoor space which is mainly for visualization, indoor navigation and Computer Aided Design (CAD). Geometric model can support representation of location and direction but it is not helpful for indoor navigation queries (Zhang et al., 2016).

Symbolic model is an indoor space model which each indoor space element has a symbolic ID. The relation between symbolic entities represents topological relationship in indoor space. Symbolic model consists of four categories including semantic-based, topology-based, lattice-based and graph-based. There are several researches based on the symbolic model ( $\mathrm{Li}$ and Lee, 2008; Jensen et al., 2009; Yang et al., 2009) which represents indoor connectivity but can hardly support geometrical features in indoor space (Zhang et al., 2016).

Object feature model is an indoor space model which represents features of indoor space and the relationship between types and operations. CityGML and IndoorGML (Kolbe et al.,2005) are examples of object feature model. Indoor space is divided to sub-space by IndoorGML (e.g. wall, roof, floor) and contain semantic information and topology in UML classes. IndoorGML cannot represent the geometrical feature of indoor elements and cannot support indoor distance-aware queries either (Zhang et al., 2016).

A Hybrid model is an indoor space model which represents a combination of previous discussed models ( $\mathrm{Li}$ et al., 2010). According to Brown et al. (2013), there is a general absence of comprehension about the necessities for topographic space data to be utilized as a part of indoor route applications and therefore the suitability of the existing data sources. In this manner there is a need to enhance the comprehension of the semantics and the limitations required for topographic space. Building models are progressively being utilized to give the topographic space data, despite the fact that these models have not been produced considering this particular application (Brown et al., 2013).

A 3D indoor network model generation is a labor-intensive process and it gets worse if some nodes require extra information (Teo and Cho, 2016). 3D buildings require new data information sources as they change over time. Data information sources generated by CAD systems are not useful for detailed indoor applications as they just roughly approximate indoor building entities. In this paper, a hybrid indoor space model is presented. In this approach, surveyed benchmarks are considered as dual node and generated 3D building model is considered as primal graph. This paper is organized as follows: Researches related to indoor path finding is discussed in section 2. Section 3 presents current 
study of indoor space modeling. Conclusion is discussed in section 4 .

\section{Indoor path finding}

For indoor navigation, GNM is vastly used due to its simplicity. GNM models are mostly extracted from 2D plans and the extracted indoor network information is coarse. GNM models do not contain accurate indoor information and they are complex and time consuming to be generated (Teo and Cho, 2016).

A 3D indoor network model generation is a labor-intensive process and it gets worse if some nodes require extra information (Teo and Cho, 2016). 3D buildings require new data information sources as they change over time. Data information sources generated by CAD systems are not useful for detailed indoor applications as they just roughly approximate indoor building entities. According to Vanclooster and Maeyer (2012), indoor navigation communities focus just on technological aspect of indoor navigation or on the generation of indoor data structure (Lee and Kwan, 2005; Lorenz et al., 2006; Richter et al., 2011).

CityGML is one of data model used for indoor building routing. CityGML is an XML-based open data model for 3D city modeling (Kolbe et al., 2009). CityGML provides information for indoor building environment in five level of detail (LOD) for a large or small city area (see Figure 1).

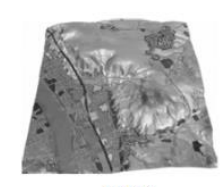

LoD0

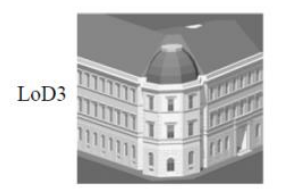

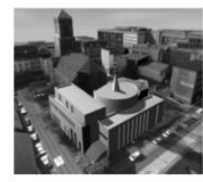

LoD2

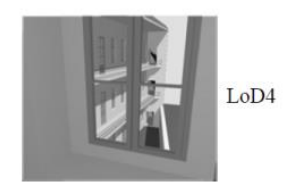

Figure 1. The five level of details (LoD) defined by CityGML (Kolbe et al., 2009).

CityGML includes geometry model and thematic model (Kim et al., 2014). CityGML has been investigated for disaster management by several researchers. Kolbe et al. (2008) investigated CityGML models for indoor building navigation. Groger and Plumer (2010) extracted graph structure with generation of CityGML models by grammar rules with semantic characterization. Becker et al. (2009) partitioned CityGML models interior to non-overlapping cells to represent topology and geometry.

Schulte and Coors (2008) used CityGML data model for flood simulation. Moreno et al. (2011) investigated application of CityGML for forest fire simulation. Most of discussed researches used GNM to represent indoor building network.
BIM is another data model used for route planning in emergency response. BIM integrates geometrical and semantic information and it can be integrated to Virtual Reality (VR) techniques (Ruppel et al., 2010). Vanclooster and Maeyer (2012) suggested that a proper indoor route planning requires indoor network, semantic information and connection between indoor and outdoor network which the integration of GIS and BIM can be a solution.

BIM provides indoor spatial information but it lacks spatial functionality (Daum and Borrmann, 2014) and GIS provides spatial information relevant to outdoor, thus the integration of BIM and GIS can be used for emergency response (Teo and Cho, 2016).

Chen and Feng (2009) presented that for a smart city application, integration of BIM and GIS for emergency response is a priority. BIM models contain detailed indoor information but they are complex. BIM has been used for safety analysis (Zhang and $\mathrm{Hu}, 2011$ ) and fire safety management (Ruppel and Schntz, 2011; Li et al., 2014).

Ruppel et al. (2010) presented a system to find shortest path for emergency rescuers in a complex building structure. Abolghasemzadeh (2013) presented a method for fire emergency response simulation considering behavioural response of people in a dynamic environment.

According to Kim et al. (2014), GIS presents real world as low-level elements (e.g. points, lines and polygon) where BIM uses high-level building elements (e.g. walls and doors) which makes the integration of GIS and BIM more complex. Generation of BIM models is labour-intensive, time consuming and with complex data structure. An important aspect forgotten or simply ignored in the most of the discussed researches is the required preliminary data for 3D building modeling. Data collection is the first aspect of any GIS system which is a costly and time consuming process.

In the most of the above discussed researches, precise and up-to-date geometrical models are vital. This research investigates to propose new methodology to overcome the need of required precise geometrical models for the derivation of topological model. 3D indoor navigation modeling requires a valid 3D model that can be represented as a cell complex: a model without any gap or intersection such that two cells (e.g. room, corridor) perfectly touch each other.

\section{A hybrid 3D indoor space model}

In a graph, nodes represent location of an object (e.g. a room) and edges represent connection between nodes. Traveling time required to move from one node to another node is an example of use of a graph. A graph $\mathrm{G}$ is defined as a finite and non-empty set of vertices $\mathrm{V}$ and a set of unordered pairs of distinct nodes (edges) $E$, i.e., $G=(V, E)$ Vertices $x$ and $y$ of graph $G$ are incident with an edge $e$ when e joins $x$ to $y$ : e=xy (Worboys and Duckham, 2004). 
Semantically rich information, automated generation of network, dynamic routing capacity and door-to-door movement capacity are a portion of the necessities in indoor route modeling (Liu and Zlatanova, 2011). Semantically-rich means the valuable data of each geometrical object, for example, area of entryways or exit points in a building. Automatic generation of network from original geometry means that navigation model needs to be automatically generated from geometrical objects. Dynamic routing means that with environment changes; navigation paths should be reconstructed. Door-to-door movement is related to the natural movement inside a building for a pedestrian.

In the proposed 3D hybrid indoor space model, surveying benchmarks are considered as dual node while the generated 3D model is represented as a primal graph. Each room, corridor and benchmark has a specific ID which is referred as a primary key. Surveying benchmarks and building model are connected in accordance with their IDs.

Semantic information such as room's number, room's name, number of control points, number of windows and other attributes can be attached to each primal graph. Semantic information is used for the recognition of two neighboring rooms or corridors. Each Primal graph contains one or several dual nodes which can be utilized in the further processes (ID of rooms and corridors will be used for the dual nodes connectivity).

The indoor navigation network modeling consists of two main procedures $-3 \mathrm{D}$ building modeling and navigation network modeling. These procedures can be further explained in five steps as follows:

1. Geometry of each room and corridor constitutes at least eight vertices along with their $\mathrm{x}, \mathrm{y}$ and $\mathrm{z}$ coordinates. These coordinate vertices for each room and corridor are as an input as in Figure 2.

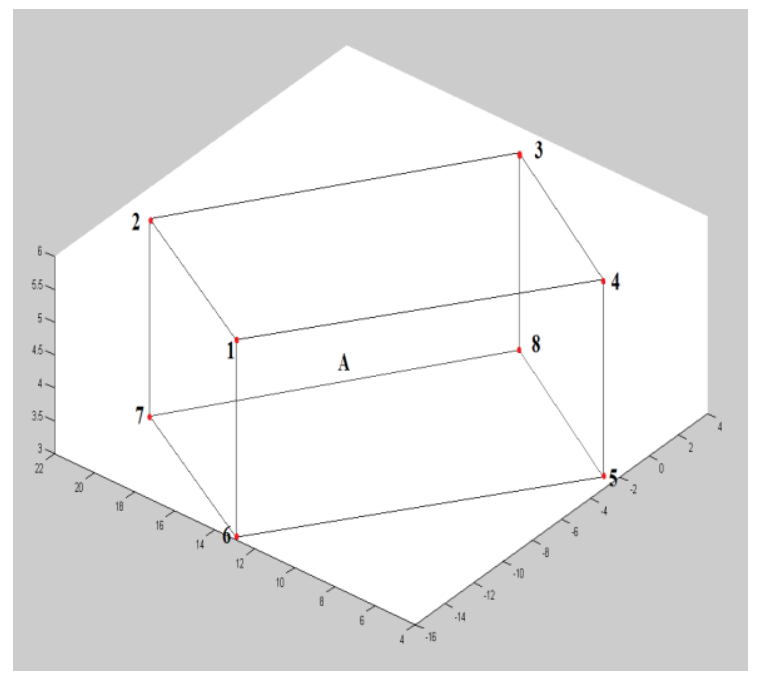

Figure 2. A room block where vertices are represented as red points.
2. Surveying benchmarks are input as control points (see Figure 3). These surveying control points belonging to doors, elevators, stairs, rooms and corridor are considered as dual nodes.

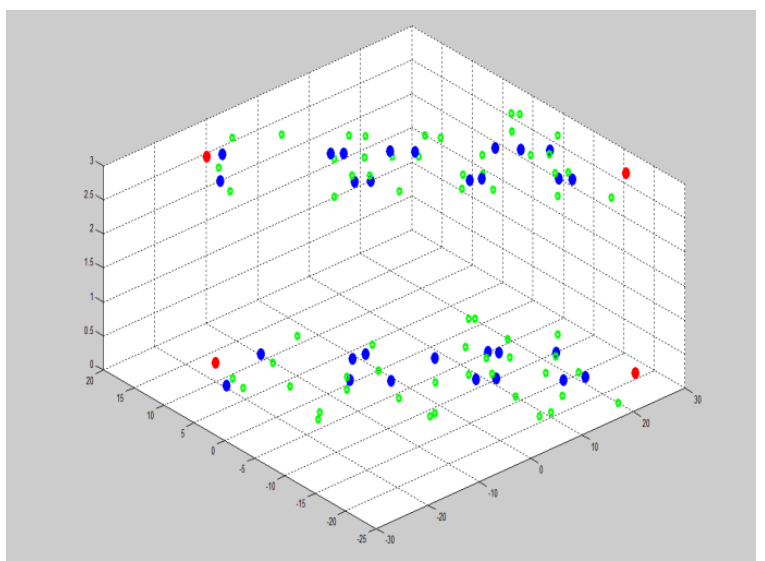

Figure 3. Surveying control points as dual nodes: doors are represented as blue points, elevators as red points while room and corridor control points as green points.

3. $3 \mathrm{D}$ modeling of indoor building environment is done based on input 3D coordinates of rooms and corridor (see Figure 4).

In this research, 3D modeling is based on boundary representation with using vertices and faces.

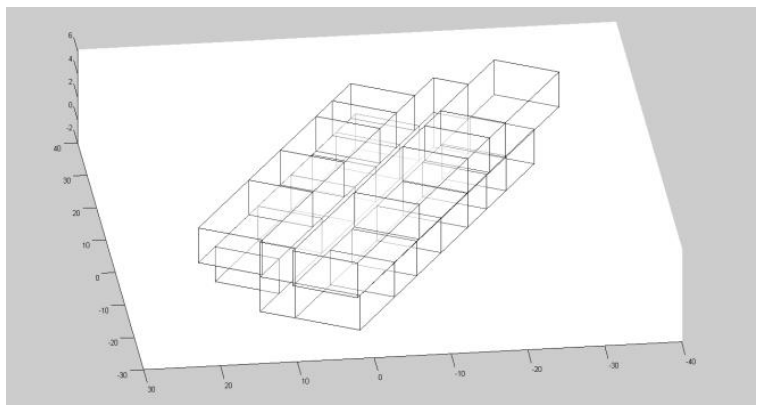

Figure 4. 3D building modeling.

4. Connection between control points.

Topological connection between control points is generated using Delaunay Triangulation. The connection of control points using Delaunay Triangulation facilities navigation and neighborhood queries along rooms and corridor network.

\section{Connection between rooms.}

In order to build connection, three different scenarios i.e. 'gap', 'intersect' and 'touch' are considered. Connection between rooms (see Figure 5) has been considered as it is important for various applications such for disaster management system and safety analysis, for example, if there is a need to break walls between two rooms. 


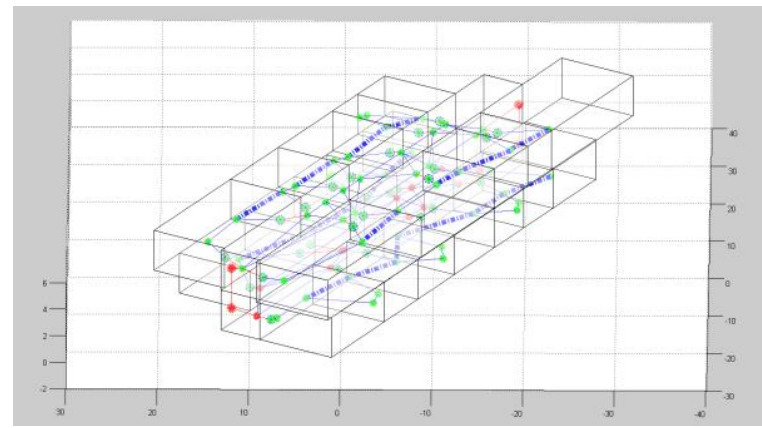

Figure 5. The proposed hybrid indoor space model where surveying control points are represented as green points, connection between dual nodes as blue lines, connection between rooms as blue dash lines and connection between floors as red lines.

To check the implemented algorithm for connection between neighboring cells, four different scenarios as test cases were designed and implemented. They have different numbers of control points and different geometrical models.

Test case 1 includes ten rooms, one corridor and fifteen control points (see Figure 6).

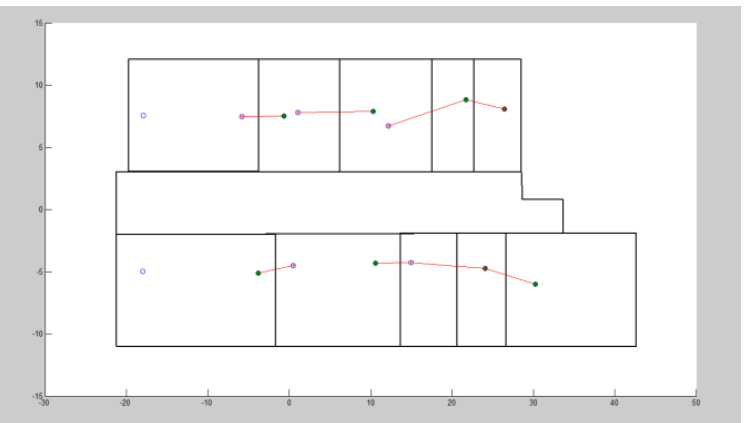

Figure 6. Test case 1 where surveying control points are represented as colored points and connection between dual nodes as red lines.

Test case 2 includes twelve rooms, one corridor and thirteen control points (see Figure 7).

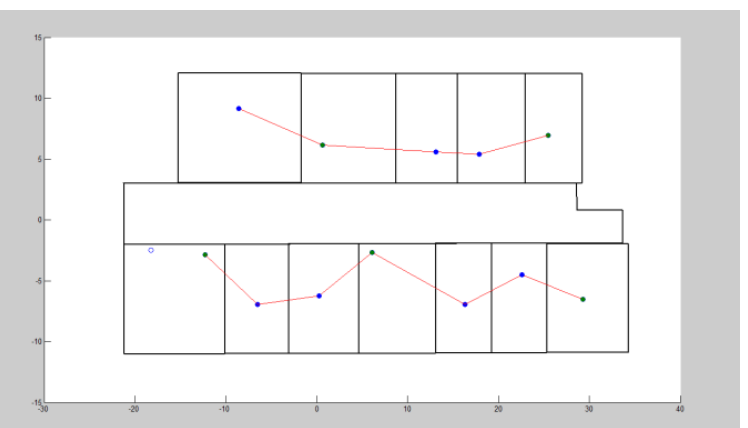

Figure 7. Test case 2 where surveying control points are represented as colored points, connection between dual nodes as red lines.
Test case 3 includes eleven rooms, one corridor and sixteen control points (see Figure 8).

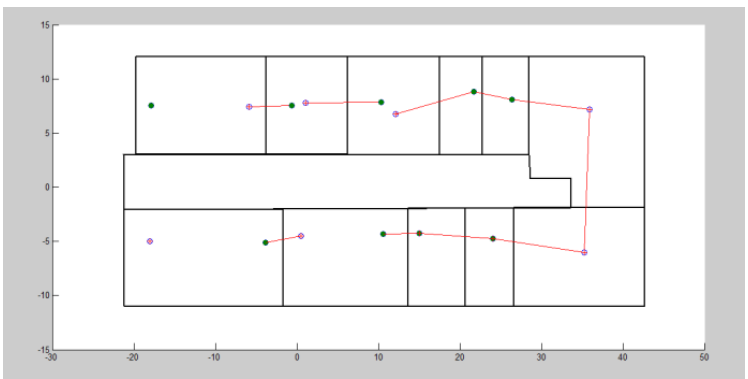

Figure 8 . Test case 3 where surveying control points are represented as colored points and connection between dual nodes as red lines.

Test case 4 includes elven rooms, one corridor and sixteen control points (see Figure 9).

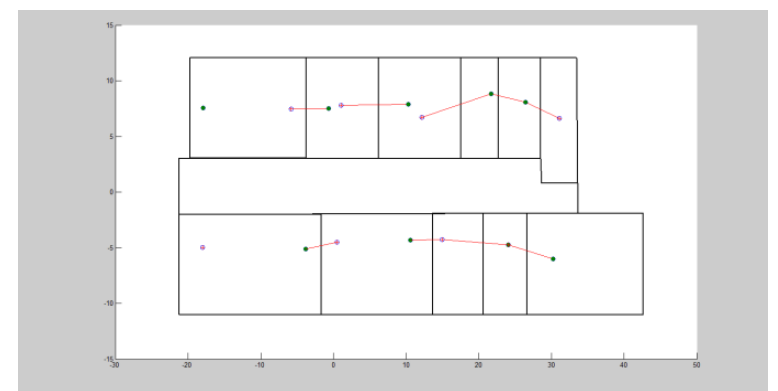

Figure 9. Test case 4 where surveying control points are represented as colored points and connection between dual nodes as red lines.

Considering the different geometrical models and different numbers of control points for each defined test case; the implemented algorithm works properly (the connections between neighboring cells were created correctly).

Validation of implemented algorithm is based on the generated connectivity of dual nodes belonging to each room (closest dual nodes for neighboring cells supposed to be connected).

Finally, for testing the implemented network, Dijkstra's algorithm (Dijkstra, 1959) is used to find shortest path between dual nodes (see Figure 10). Dijkstra's algorithm is an algorithm to find shortest path between two nodes in a graph (e.g. location of a person to nearest exit point inside a building). 
a)

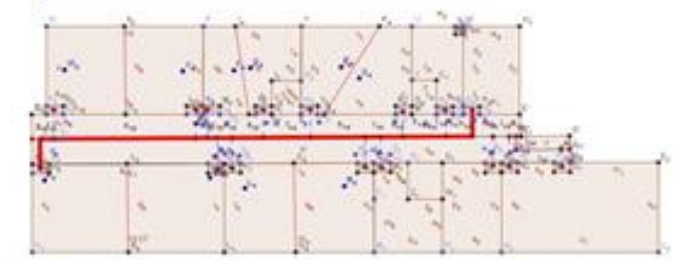

b)

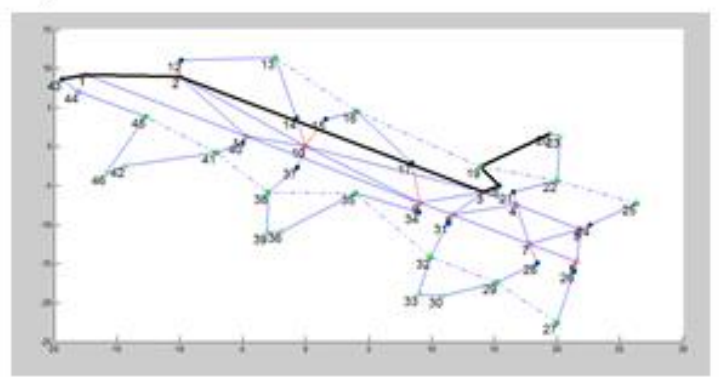

Figure 10. Dijkstra's algorithm for shortest path finding where a) red line represents optimal route between two dual nodes b) black line represents optimal route between two dual nodes while corridor is divided into several regions (polygonization).

As explained in this section, the proposed hybrid 3D indoor space model consists of topological, semantic and geometrical spaces. Connectivity of dual nodes represents topological space, 3D building model represents geometrical space and attributes attached to each building entity (e.g. door) shows semantic space.

\section{Conclusion}

In this paper, a 3D hybrid indoor space model has been presented. The presented topological model uses semantic and geometrical information for topological connectivity. This research attempts to propose a new methodology for indoor space modeling for buildings whiteout proper 2D/3D geometrical models. A proper model for navigation requires topology, geometry and semantic information. Geometry and topology are defined by dual and primal spaces. Constraints of a model (e.g. obstacles) and material of building elements (e.g. glass doors) can be defined and be attached to building models by semantic information.

\section{References}

Abolghasemzadeh, P. (2013). A comprehensive method for environmentally sensitive and behavioral microscopic egress analysis in case of fire in buildings. Safety science, $59,1-9$.

Becker, T., Nagel, C., \& Kolbe, T. H. (2009). A multilayered space-event model for navigation in indoor spaces. In $3 D$ Geo-Information Sciences (pp. 61-77). Springer Berlin Heidelberg.
Boguslawski, P., Gold, C.M. and Ledoux, H., 2011. Modeling and analysing 3D buildings with a primal/dual data structure. ISPRS Journal of Photogrammetry and Remote Sensing, 66(2): 188-197.

Brown, G., Nagel, C., Zlatanova, S., \& Kolbe, T. H. (2013). Modeling 3D topographic space against indoor navigation requirements. In Progress and New Trends in $3 D$ Geoinformation Sciences (pp. 1-22). Springer Berlin Heidelberg.

Dijkstra, E. W. (1959). A note on two problems in connexion with graphs. Numerische mathematik, 1(1), 269271.

Gröger, G., \& Plümer, L. (2010). Derivation of 3D indoor models by grammars for route planning. PhotogrammetrieFernerkundung-Geoinformation, 2010(3), 191-206.

Jensen, C. S., Lu, H., \& Yang, B. (2009, May). Graph model based indoor tracking. In Mobile Data Management: Systems, Services and Middleware, 2009. MDM'09. Tenth International Conference on (pp. 122-131). IEEE.

Kim, J. S., Yoo, S. J., \& Li, K. J. (2014, May). Integrating IndoorGML and CityGML for indoor space. In International Symposium on Web and Wireless Geographical Information Systems (pp. 184-196). Springer Berlin Heidelberg.

Kolbe, T. H., Gröger, G., \& Plümer, L. (2005). CityGML: Interoperable access to 3D city models. In Geo-information for disaster management (pp. 883-899). Springer Berlin Heidelberg.

Kolbe, T. H. (2009). Representing and exchanging 3D city models with CityGML. In $3 D$ geo-information sciences (pp. 15-31). Springer Berlin Heidelberg.

Lee, J., \& Kwan, M. P. (2005). A combinatorial data model for representing topological relations among 3D geographical features in micro-spatial environments. International Journal of Geographical Information Science, 19(10), 1039-1056.

Li, D., \& Lee, D. L. (2008, April). A lattice-based semantic location model for indoor navigation. In Mobile Data Management, 2008. MDM'08. 9th International Conference on (pp. 17-24). IEEE.

Li, X., Claramunt, C., \& Ray, C. (2010). A grid graphbased model for the analysis of $2 \mathrm{D}$ indoor spaces. Computers, Environment and Urban Systems, 34(6), 532540.

Liu, L., \& Zlatanova, S. (2011). Towards a 3D network model for indoor navigation. Urban and Regional Data Management, UDMS Annual, 79-92.

Lorenz, B., Ohlbach, H. J., \& Stoffel, E. P. (2006). A hybrid spatial model for representing indoor environments. 
In Web and Wireless Geographical Information Systems (pp. 102-112). Springer Berlin Heidelberg.

Moreno, A., Segura, Á., Korchi, A., Posada, J., \& Otaegui, O. (2011). Interactive urban and forest fire simulation with extinguishment support. In Advances in $3 D$ GeoInformation Sciences (pp. 131-148). Springer Berlin Heidelberg.

Richter, K. F., \& Duckham, M. (2008, September). Simplest instructions: Finding easy-to-describe routes for navigation. In International Conference on Geographic Information Science (pp. 274-289). Springer Berlin Heidelberg.

Rüppel, U., Abolghasemzadeh, P., \& Stübbe, K. (2010, June). BIM-based immersive indoor graph networks for emergency situations in buildings. In International Conference on Computing in Civil and Building Engineering (ICCCBE).

Schulte, C., \& Coors, V. (2008, August). Development of a CityGML ADE for dynamic 3D flood information. In Joint ISCRAM-CHINA and GI4DM Conference on Information Systems for Crisis Management.

Teo, T. A., \& Cho, K. H. (2016). BIM-oriented indoor network model for indoor and outdoor combined route planning. Advanced Engineering Informatics, 30(3), 268282.

Vanclooster, A., \& De Maeyer, P. (2012). Combining indoor and outdoor navigation: the current approach of route planners. In Advances in Location-Based Services (pp. 283-303). Springer Berlin Heidelberg.

Worboys, M. F., \& Duckham, M. (2004). GIS: a computing perspective. CRC press.

Yang, B., Lu, H., \& Jensen, C. S. (2009, November). Scalable continuous range monitoring of moving objects in symbolic indoor space. In Proceedings of the 18th ACM conference on Information and knowledge management (pp. 671-680). ACM.

Zhang, X., Jin, P., Yue, L., Wang, N., \& Li, Q. (2016). Towards Mobile Information Systems for Indoor Space. Mobile Information Systems. 